\title{
Parallel Pathways for Cross-Modal Memory Retrieval in Drosophila
}

\author{
Xiaonan Zhang, ${ }^{1,3}$ Qingzhong Ren, ${ }^{2}$ and Aike Guo ${ }^{1,2}$ \\ ${ }^{1}$ State Key Laboratory of Brain and Cognitive Science, Institute of Biophysics, Chinese Academy of Sciences (CAS), Beijing 100101, China, ${ }^{2}$ Institute of \\ Neuroscience, State Key Laboratory of Neuroscience, Shanghai Institutes for Biological Sciences, CAS, Shanghai 200031, China, and ${ }^{3}$ University of CAS, \\ Beijing 100049, China
}

\begin{abstract}
Memory-retrieval processing of cross-modal sensory preconditioning is vital for understanding the plasticity underlying the interactions between modalities. As part of the sensory preconditioning paradigm, it has been hypothesized that the conditioned response to an unreinforced cue depends on the memory of the reinforced cue via a sensory link between the two cues. To test this hypothesis, we studied cross-modal memory-retrieval processing in a genetically tractable model organism, Drosophila melanogaster. By expressing the dominant temperature-sensitive shibire ${ }^{t s l}\left(s h i^{t s I}\right)$ transgene, which blocks synaptic vesicle recycling of specific neural subsets with the Gal4/ $U A S$ system at the restrictive temperature, we specifically blocked visual and olfactory memory retrieval, either alone or in combination; memory acquisition remained intact for these modalities. Blocking the memory retrieval of the reinforced olfactory cues did not impair the conditioned response to the unreinforced visual cues or vice versa, in contrast to the canonical memory-retrieval processing of sensory preconditioning. In addition, these conditioned responses can be abolished by blocking the memory retrieval of the two modalities simultaneously. In sum, our results indicated that a conditioned response to an unreinforced cue in cross-modal sensory preconditioning can be recalled through parallel pathways.
\end{abstract}

\section{Introduction}

To survive in dynamic environments, animals must adjust their behavior by assessing risks according to multi-modal signal combinations. Thus, the ability to master object associations between different modalities is crucial. Using the sensory preconditioning (SP) paradigm, we investigated cross-modal conditioning in Drosophila. In the SP paradigm (Brogden, 1939), a pair of conditioned stimuli (CS1 and CS2) is presented without reinforcement during the pretraining session. Then, CS2 is reinforced, either negatively or positively, by the unconditioned stimulus (US, e.g., food as reward or electric shock as punishment). Finally, the conditioned response to CS1 is observed. The conditioned response to CS1 has been observed in both vertebrates and invertebrates; CS1 and CS2 can use either the same (Müller et al., 2000;

\footnotetext{
Received Sept. 28, 2012; revised April 7, 2013; accepted April 8, 2013.

Author contributions:X.Z. and A.G. designed research;X.Z. and Q.R. performed research; X.Z. analyzed data; X.Z., Q.R., and A.G. wrote the paper.

This work was supported by 973 Program (Grant 2011 CBA00400 to A.G.), the National Science Foundation of China (Grants 30921064, 90820008, and 31130027 to A.G.), and the "Strategic Priority Research Program" of the Chinese Academy of Sciences (Grant XDB02040100) to A.G. The funders had no role in the study design, data collection and analysis, the decision to publish, or the preparation of this manuscript. We thank Xing Yang and Ke Chen for their assistance with the experiments; Mingkui Zhang and Jingwu Hou for their technical assistance; and Ronald Davis, Li Liu, Tim Tully, Martin Heisenberg, the Bloomington Stock Center, and the Drosophila Genetic Resource Center for generously providing fly stocks and reagents. We also thank the anonymous reviewers for their thoughtful comments, which greatly improved our manuscript.

The authors declare no competing financial interests.

Correspondence should be addressed to Dr. Aike Guo, Institute of Neuroscience, State Key Laboratory of Neuroscience, Shanghai Institutes for Biological Sciences, Chinese Academy of Sciences, 320 Yue Yang Road, Shanghai 200031, China.E-mail:akguo@ion.ac.cn.

Q. Ren's present address: Janelia Farm Research Campus, Howard Hughes Medical Institute, Ashburn, VA 20176 DOI:10.1523/JNEUROSCI.4631-12.2013

Copyright $\odot 2013$ the authors $\quad 0270-6474 / 13 / 338784-10 \$ 15.00 / 0$
}

Brembs and Heisenberg, 2001) or different sensory modalities (Tait et al., 1972; Hall and Suboski, 1995; Kojima et al., 1998; Guo and Guo, 2005; Matsumoto et al., 2013).

The CS1-CS2 association was thought to be vital in the memory-retrieval processing of CS1 (Gewirtz and Davis, 2000) because only CS2 was reinforced during the learning tasks. Thus, the prevailing hypothesis for the memory retrieval of SP is that the conditioned response to CS1 is dependent on the CS2 memory via a CS1-CS2 association. Some regions of the limbic system (e.g., the perirhinal cortex and the retrosplenial cortex) that receive information from multiple sensory modalities were found to be necessary for CS1-CS2 association acquisition in the rat (Nicholson and Freeman, 2000; Robinson et al., 2011). However, neither the pathways nor the underlying neural circuits for retrieving the conditioned response to CS1 are well understood.

In the present study, we established a modified cross-modal (olfaction and vision) SP paradigm and showed that flies exhibited robust conditioned responses to the unreinforced cues (CS1) during the test. Olfactory and visual short-term memory processes have been reported to be anatomically separated in flies (Zars, 2010). Therefore, using a genetic silencer of synaptic transmission, we specifically blocked the memory retrieval of olfaction and vision, either alone or simultaneously. If the conditioned response to CS1 can be recalled only through a CS1-CS2 association, the CS1 memory would be abolished once the memory retrieval of CS2 was fully disrupted, unless there is another pathway for CS1 memory retrieval. Our results revealed parallel pathways and neural circuits for cross-modal memory retrieval, suggesting that more than one memory trace is formed in the SP paradigm. 
A



B
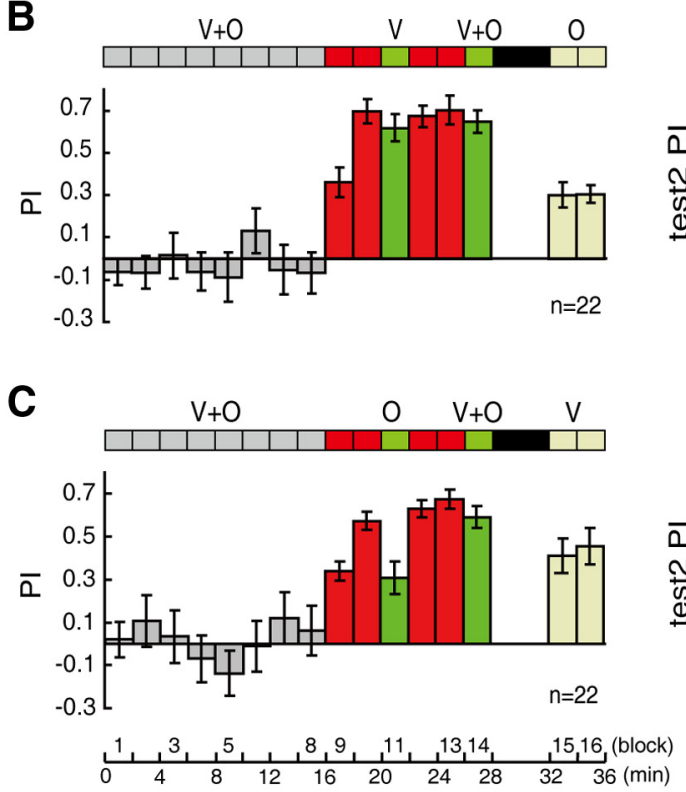

D



E panorama for cross-modal Vision (V) and Olfaction (O)



panorama for Blank (B) or only Olfaction (O)

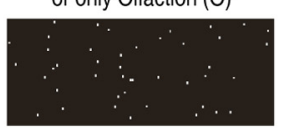

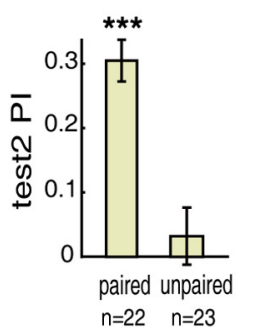

$\square$ Pre-training

$\square$ Training

$\square$ Test 1

$\square$ Test 2

Dark
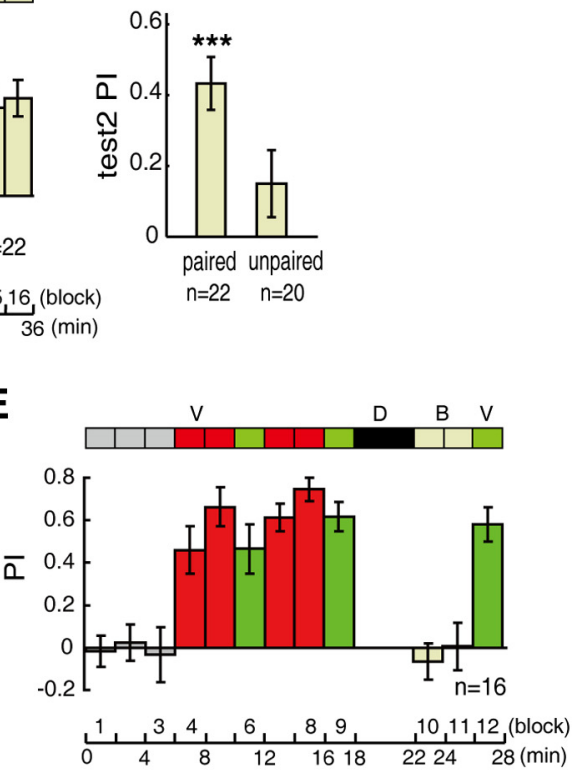

Figure 1. Cross-modal sensory preconditioning. $\boldsymbol{A}$, Schematic diagram of the experimental setup. Tethered flies can control their orientations relative to visual cues or odors by generating yaw torque, which was measured with a torque meter and used to control the panorama rotation. The visual cues were horizontal bars with $30^{\circ}$ separation of elevation. The odors were $0.99 \%$ 3-octanol (OCT) and 0.99\% 4-methylcyclohexanol (MCH). B, Top, Time course of cross-modal SP. Individual Canton-S flies were subjected to paired visual and olfactory cues in the pretraining session $(\mathrm{V}+0)$, followed by unimodal visual conditioning $(\mathrm{V})$. The paired cues were presented to the flies again as a reminder, and the olfactory cues $(0)$ were tested after a 4 min dark period. The Test $2 \mathrm{PI}$ (averaged in the right panel) was significantly positive $(p<0.001)$ after the visual and olfactory cues were explicitly paired in time, but was not significantly different from zero after presenting the unpaired cues. $\boldsymbol{C}$, Similar to $\boldsymbol{B}$, but flies were trained with olfaction and tested with vision. $\boldsymbol{D}$, The random spots did not interfere with olfactory conditioning. The flies were subjected to normal odor delivery, no odor, or reversed odor delivery in the last 6 min of the protocol. Top, Time course of unimodal olfactory conditioning. Bottom, The averaged Pls of the Test 2 section (block 10-12). $\boldsymbol{E}$, The random spots did not interfere with visual conditioning. The flies were subjected to a 4 min dark interval ( $D$, dark) followed by a 4 min period with no visual cues ( $B$, blank) and visual cues (V) again, in the test session. Data are presented as means \pm SEM. $n$ indicates the total number of flies examined. ${ }^{* * *} p \leq 0.001$.

\section{Materials and Methods}

Flies and preparation. The (247,c205)-Gal4 line was generated by 247 and c205 recombination. The $247>\operatorname{sh} i^{t s 1}, \mathrm{c} 205>\operatorname{sh} i^{t s 1},(247, \mathrm{c} 205)>\operatorname{sh} i^{t s 1}$,

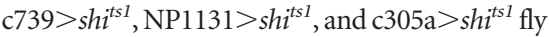
strains were generated by crossing the Gal4 drivers 247, c205, (247,c205), c739, NP1131, and c305a, respectively, with UAS-shits ${ }^{t s 1}$ The heterozygotes $247 /+$, c205/+, $(247, \mathrm{c} 205) /+$, $\mathrm{c} 739 /+, \mathrm{NP} 1131 /+, \mathrm{c} 305 \mathrm{a} /+$, and $s h i^{t s l} /+$ were generated by crossing the Gal4 or UAS lines with the wild-type strain Canton-S. The NP1131 and c305a-Gal4 strains were obtained from the Drosophila Genetic Resource Center (Kyoto Institute of Technology, Kyoto, Japan) and the Bloomington Stock Center (Indiana University, Bloomington, IN), respectively. UAS-shi $i^{t s l}$ is dominant temperature sensitive and blocks synaptic vesicle recycling at the restrictive temperature (RT) (Kitamoto, 2001); this fly strain was kindly provided by Dr. T. Tully (Dart Neuroscience LLC, San Diego, CA). The following fly strains were also kind gifts: 247-Gal4 from Dr. M. Heisenberg (University of Würzburg, Würzburg, Germany); c205Gal4 from Dr. L. Liu (Institute of Biophysics, Chinese Academy of Sciences); and c739-Gal4 from Dr. R. Davis (The Scripps Research Institute, Jupiter, FL). All flies were cultured at $25^{\circ} \mathrm{C}$ and $60 \%$ humidity on standard medium under a $12 \mathrm{~h}$ light/12 h dark cycle (Guo et al., 1996).

Three- to five-day-old adult female flies were briefly immobilized by cold anesthesia, and a tiny triangle-shaped hook (diameter 0.05 $\mathrm{mm}$ ) was glued to the dorsal thorax and head. The flies were then kept individually in small chambers for overnight recovery and were fed with sucrose solution.

The apparatus. A single, tethered fly was suspended at a torque meter (Götz, 1964) in the center of an arena (Fig. $1 A$ ), which was illuminated from behind. The yaw torque generated by the fly (detected by the torque meter) drove the rotation of the arena via a negative feedback mechanism (Wolf and Heisenberg, 1991). The apparatus was designed to stabilize the fly so it could choose its flight orientation with respect to the arena by adjusting its yaw torque. The panorama of the arena was virtually divided into four quadrants, and $2 \%$ of the area was randomly filled with white dots (view angle $<$ $2.5^{\circ}$; Fig. $1 A$ ). The visual and olfactory cues were presented in alternating sequence in each quadrant (Fig. 1A). The visual cues consisted of two upper and two lower white horizontal bars $\left(40^{\circ} \times 12^{\circ}\right)$ and were defined by their center of gravity separation. The olfactory cues consisted of $0.99 \% 3$-octanol (OCT) and 0.99\% 4-methylcyclohexanol (MCH), which were presented by an air delivery system (Guo and Guo, 2005). For conditioning, one of the two cues was paired with a noxious heat stimulus ( $\mathrm{CO}_{2}$ laser beam, $\left.\lambda=10600 \mathrm{~nm}\right)$. During the sessions before and after training, the heat was switched off.

Behavioral experiments. The conditioning protocol involved three or four temporal sessions (denoted by colored blocks in the figures): pretraining (gray), training (red), Test 1 (light green), and Test 2 (light yellow). Be- 


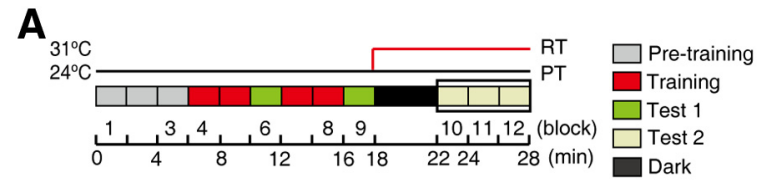

B


Figure 2. $\mathrm{MB}$ and $\mathrm{F} 5$ output are necessary for olfactory and visual memory retrieval, respectively. $\boldsymbol{A}$, Time course of unimodal olfactory or visual conditioning. The flies were tested at either the RT (red line) or the PT (black line). The mean PIs in the Test 2 section of unimodal conditioning (block 10-12) are analyzed below. B, Blocking the synaptic output from the 247-Gal4 neurons impaired the olfactory memory-retrieval process (left) but not the visual memoryretrieval process (right) at the RT. C, Blocking the synaptic output from the $205-G a l 4$ neurons impaired the visual elevation memory retrieval (left) but not the olfactory memory retrieval (right) at the RT. The flies were trained and tested under either unimodal olfactory or visual conditions, as highlighted by schematic diagrams ("MCH vs OCT" or bars with different elevations) above the histograms. The test PIs at the RT are represented by red rectangles, while the test PIs at the PT are indicated by black rectangles. Error bars are SEM. ${ }^{* * *} p \leq 0.001$.

tween the different stages of the test section, the arena lights were temporarily switched off (dark period, black block). The ambient temperature in the arena could be adjusted from $24^{\circ} \mathrm{C}$ [permissive temperature $(\mathrm{PT})]$ to $31^{\circ} \mathrm{C}(\mathrm{RT})$ using a feedback temperature controller unit (Zhang et al., 2007) during the dark period or could be kept stable at PT for the rest of the experiment. The dark period lasted $4 \mathrm{~min}$.

The session length changed for different behavioral paradigms. The cross-modal SP paradigm consisted of a 16 min pretraining session, a 10 min training session (including two consecutive 4 min training cycles spaced by a 2 min test block), a 2 min reminder Test 1 session, a 4 min dark period, and a $4-8$ min Test 2 session. In the pretraining session, cues of different modalities were presented to the fly as fixed pairs. Then, cues of a single modality were conditioned in the training section; a reminder block followed in which the fixed pairs of cues were presented again without punishment. Finally, the conditioned response to the cues of the other modality, which was not presented during the training section, was assessed.

The unimodal conditioning paradigm consisted of a 6 min pretraining session, a 10 min training session, a 2 min Test 1 section followed by a 4 min dark period, and a 6 min Test 2 section (see Figs. $1 D, E, 2,4 B, D, F$ ). Cues of only one modality were presented and conditioned during the unimodal conditioning paradigm.

Data analyses. The flight orientation (panorama position) was recorded and stored every $50 \mathrm{~ms}$ by a computer; this information was used to calculate the time spent in each quadrant. During the training session, the heat was switched on whenever the flight orientation fell into the dangerous quadrants and was switched off whenever the flight orienta- tion fell into the safe quadrants. The performance index (PI) was used to quantify the choice between the safe quadrants and the dangerous quadrants in each 2 min block. It was defined as $(t 1-t 2) /(t 1+t 2)$, where $t 1$ is the time spent flying toward the safe quadrants and $t 2$ is the time spent flying toward the dangerous quadrants. Thus, $\mathrm{PI}=1$ indicates that the fly spent the entire time flying toward the safe quadrants, while PI $=0$ means that the fly spent equal time flying toward the safe and dangerous quadrants. Each of the two cues was presented in the safe quadrants in half of the experiments, which eliminates the effects of spontaneous cue preferences.

Cue discrimination $(D)$ ability was derived from the flies' spontaneous cue preferences. The flight orientation with reference to a specific cue occurs at a periodicity of $180^{\circ}$, due to the alternating sequence of the equally spaced cues in the panorama (Fig. $1 \mathrm{~A}$ ). Thus, the $180^{\circ}$ periodicity of the flight orientation histogram indicates a fly's cue discrimination ability. $D$ is defined as $2 A_{180} /\left(A_{120}+A_{72}\right)$ (Liu et al., 2006), where the $180^{\circ}$ periodicity $\left(A_{180}\right)$ is considered a cue's "recognition" and the $120^{\circ}$ and $72^{\circ}$ periodicities $\left(A_{120}\right.$ and $\left.A_{72}\right)$ are considered a cue's "background." These periodicities are measured as the absolute amplitude in the Fourier spectra. When $D$ is 1 , the flies perform toward the cues and background equally. When $D$ is significantly larger than 1 , the flies exhibited normal cue discrimination ability.

Immunohistochemistry and confocal microscopy. The brains of female flies with Gal4-driven UAS-mCD8-GFP expression were dissected 3 to $6 \mathrm{~d}$ after eclosion. The immunolabeling procedure followed the standard protocol (Wu and Luo, 2006), using nc82 (1:100; Developmental Studies Hybridoma Bank) as the primary antibody and Alexa Fluor-568 goat anti-mouse (1:100; A11031, Invitrogen) as the secondary antibody.

Serial optical sections were acquired with a confocal microscope (A1R on FN1, Nikon) equipped with an objective (NIR Apo $40 \times / 0.80 \mathrm{w}$, Nikon). The sections were acquired at $1.5 \mu \mathrm{m}$ intervals with $512 \times 512$ pixel resolution. The images were then processed using ImageJ (National Institutes of Health, Bethesda, MD).

Statistical analyses. The Shapiro-Wilk's test was performed as a test of normality. For the normally distributed data, the one-sample $t$ test (twosided $p$ value) was used to determine the significance of the values of PI or $D$ (Liu et al., 1999; Brembs and Wiener, 2006; Liu et al., 2006). The two-sample $t$ test was used for comparisons between groups. Comparisons between multiple groups were conducted by a one-way ANOVA followed by a Bonferroni test. For data that were not distributed normally, nonparametric tests, including the Mann-Whitney test and the Kruskal-Wallis test, were used. Statistical analyses were performed using SPSS Statistics 17 (SPSS). The sample size of each group and the significance levels of the tests (including post hoc tests) are shown in the figures. The data are presented as means \pm SEM. Asterisks indicate levels of significance $\left({ }^{*} p \leq 0.05 ;{ }^{* *} p \leq 0.01 ;{ }^{* * *} p \leq 0.001\right)$.

\section{Results}

\section{Cross-modal SP in Drosophila}

To investigate cross-modal memory-retrieval processing in Drosophila, we modified the SP paradigm slightly from our previous study (Guo and Guo, 2005). In the pretraining session (16 min), single flies were presented with fixed pairs of visual and olfactory cues (e.g., the upper horizontal bars were always combined with $\mathrm{MCH}$ and the lower bars were combined with OCT) (Fig. $1 A$; see Materials and Methods). The cues of a single modality (e.g., only the visual modality, Fig. $1 B$ ) were then conditioned in the training session (10 $\mathrm{min}$ ), during which the flight orientation toward one set of patterns (e.g., the upper horizontal bars) was coupled with an undesirable heat punishment, while the other set (the lower bars) was not. Then, the flies were given a reminder period ( $2 \mathrm{~min}$ ) with the same pairs of cues as in the pretraining section. The choice between the cues of the other modality, which were not presented to the flies in the training section, was finally tested ( $4 \mathrm{~min}$ ) after a dark period ( $4 \mathrm{~min}$ ). If the flies learned to transfer the memory between modalities, they spent more time flying 
Table 1. Visual or olfactory cue discrimination capacity was normal in lines deficient in memory retrieval

\begin{tabular}{|c|c|c|c|c|c|}
\hline Line & $D$ value & Significance & Parameter & $n$ & $p$ value \\
\hline c205>shits ${ }^{i t}(\mathrm{RT})$ & $2.42 \pm 0.43$ & $* *$ & Visual cues (elevation) & 20 & 0.003 \\
\hline $247>\operatorname{shi}^{i s 1}(\mathrm{RT})$ & $1.89 \pm 0.35$ & * & Olfactory cues (MCH vs OCT) & 20 & 0.016 \\
\hline $\mathrm{CS}(\mathrm{PT})$ & $1.20 \pm 0.21$ & n.s. & Background (random dots) & 12 & 0.344 \\
\hline
\end{tabular}

Discrimination values $(D)$ were calculated as $D=2 A_{180} /\left(A_{120}+A_{72}\right)$. For the definition of $D$, see the Materials and Methods. The data are presented as means \pm SEM; ${ }^{*} p \leq 0.05$; ${ }^{* *} p \leq 0.01$ (one-sample $t$ test).

toward the object (e.g., the OCT) that had been associated with the safe pattern (the lower bar) of the other modality.

As in our previous study (Guo and Guo, 2005), wild-type flies were subjected to two SP subsequences: training with visual cues followed by olfactory cue testing (vision $\rightarrow$ olfaction) (Fig. 1B) and training with olfactory cues followed by visual cue testing (olfaction $\rightarrow$ vision) (Fig. 1C). Robust conditioned responses to the unreinforced cues were observed in both SP subsequences, as indicated by the significantly positive test PIs (one-sample $t$ test, $\left.t_{(21)}=9.357, p<0.001 ; t_{(21)}=5.796, p<0.001\right)$ (Fig. $1 B, C$, right panels). Importantly, the significant conditioned response was not a second-order conditioning effect (Tabone and de Belle, 2011) caused by the reminder block. The presentation of unpaired cues in the pretraining section failed to result in the memory transfer, as indicated by the test PIs, which were not significantly different from zero (one-sample $t$ test, $t_{(22)}=0.725$, $p=0.476 ; t_{(19)}=1.585, p=0.129$ ) (Fig. $1 B, C$, right panels).

We performed several experiments to exclude the potential influence of the random-dots background (Fig. 1A) on conditioning or memory retrieval. The random dots were added to eliminate the potential light-dark contextual influence of the switch between the visual cues and odor presentation. The use of random dots may also support the establishment of a coherent environment for stabilizing the flight orientation when odors were presented alone (Duistermars and Frye, 2008). In the unimodal olfactory conditioning paradigm (Fig. $1 D$ ), wild-type flies were exposed to normal odor, no odor, and reversed odor delivery conditions in the test session. When the odor delivery was turned off, the flies failed to produce significantly positive PIs in the presence of the background only (one-sample $t$ test, $t_{(11)}=$ $-1.846, p=0.092$ ) (Fig. $1 D$, middle). When the odor delivery was reversed, flies learned to avoid the dangerous odor but not the dangerous quadrant, as indicated by the significantly negative PI (one-sample $t$ test, $t_{(11)}=-7.110, p<0.001$ ) (Fig. $1 D$, right). In the unimodal visual conditioning paradigm (Fig. $1 E$ ), the test PI remained at the same level after a 4 min dark interval and 4 min exposure to the random-dots background alone (PIs of block 9 vs block 12; paired-samples $t$ test, $\left.t_{(15)}=0.472, p=0.644\right)$. The random-dots background itself did not evoke any significantly positive PIs (PIs of block 10 and 11: one-sample $t$ test, $t_{(15)}=-0.780$, $\left.p=0.448 ; t_{(15)}=0.062, p=0.952\right)$. Thus, the random-dots background itself did not interfere with either CS conditioning or memory retrieval.

\section{Blocking mushroom bodies and layer 5 of the fan-shaped body abolishes the respective short-term memory retrieval of olfaction and vision}

To dissect the memory-retrieval processing of the cross-modal SP paradigm, we identified mechanisms by which to specifically abolish the memory retrieval of unimodal cues (Fig. 2 A). In Drosophila, the memory trace for olfactory conditioning is thought to mainly reside in the mushroom bodies (MBs) (Heisenberg, 2003; Krashes et al., 2007; Wang et al., 2008a; Davis, 2011), which contain 2500 Kenyon cells in each brain hemisphere. The visual elevation memory trace is found in layer 5 (F5) of the fan-shaped body (Liu et al., 2006; Wang et al., 2008b), which is the largest component of the central complex in Drosophila.

To investigate the function of MBs in the memory-retrieval process of the unimodal conditioning task, we blocked the output of the MBs via expression of the dominant temperature-sensitive shibire ${ }^{t s l}\left(s h i^{t s l}\right)$ transgene at RT (Kitamoto, 2001) in 247-Gal4. In the unimodal conditioning paradigm (Fig. 2A), 247>shits impaired the memory retrieval of olfactory conditioning at RT (Kruskal-Wallis test, $H_{(4)}=40.513, p<0.001$ ) (Fig. $2 B$, left). Olfactory memory retrieval was totally abolished, as indicated by the lack of a statistical difference between the test PI and zero (one-sample $t$ test, $t_{(19)}=1.663, p=0.113$ ). In addition, the $247 /+$ and $s h i^{t s 1} /+$ flies exhibited normal memory retrieval at RT $\left(31^{\circ} \mathrm{C}\right)$, indicating that the impairment was not due to the RT. In contrast, $247>s h i^{t s 1}$ did not impair visual memory retrieval at RT (two-sample $t$ test, $t_{(29)}=0.751, p=0.459$ ) (Fig. $2 B$, right).

To investigate the function of F5, we chose the c205-Gal4 line (Liu et al., 2006) to block the output of F5 via expression of $s h i^{t s 1}$. At RT, c205>shits impaired visual elevation memory retrieval (ANOVA, $F_{(4,74)}=23.591, p<0.001$ ) (Fig. $2 C$, left) but left the olfactory memory retrieval intact (two-sample $t$ test, $t_{(20)}=$ $0.967, p=0.345$ ) (Fig. $2 C$, right). Again, the impairment caused a PI that was not significantly different from zero (one-sample $t$ test, $t_{(19)}=-1.719, p=0.102$ ). Furthermore, the flies with low test PIs maintained significant cue discriminability (Table 1; see Materials and Methods). Thus, these results suggest that blocking the outputs of MBs and F5 specifically abolishes the olfactory and elevation memory-retrieval processes, respectively.

\section{The memory of cross-modal SP can be retrieved through parallel pathways}

The double dissociation of separated neurons for olfactory and visual memory retrieval offered us an opportunity to discern the neural underpinnings of the memory-retrieval process in the cross-modal SP paradigm. In the olfaction $\rightarrow$ vision subsequence, the olfactory cues were reinforced, and the response to visual cues was tested. First, we blocked the output of the MBs in the test section to determine whether the flies could recall the memory of the visual modality (CS1) after the olfactory memory (CS2-US) was blocked. Thus, the $247>s h i^{t s 1}$ flies were trained at the PT but tested at either the PT or RT in the olfaction $\rightarrow$ vision subsequence. Strikingly, we found that the conditioned response to the visual stimuli was not impaired by blocking the synaptic output from the MBs (two-sample $t$ test, $t_{(33)}=1.681, p=0.102$ ) (Fig. 3A), in contrast to the CS1-CS2-US assumption, which predicts that memory recall through CS2-US would be disrupted. Therefore, association between the two CS is not necessary during memory retrieval. This finding also suggests that another memory trace is formed during conditioning (e.g., a direct CS1-US association).

The CS1 that elicits the conditioned response can be either visual or olfactory. Therefore, we designed a totally symmetrical protocol to test the potentially formed CS1 memory trace by disrupting visual memory retrieval (CS2-US) in the vision $\rightarrow$ olfaction subsequence. We blocked the F5 output during the test 
A ${ }^{310 \mathrm{C}}$
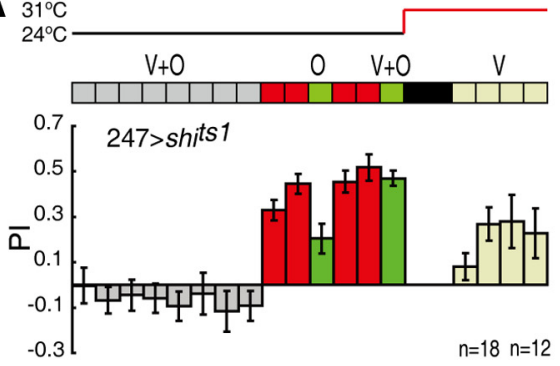

C
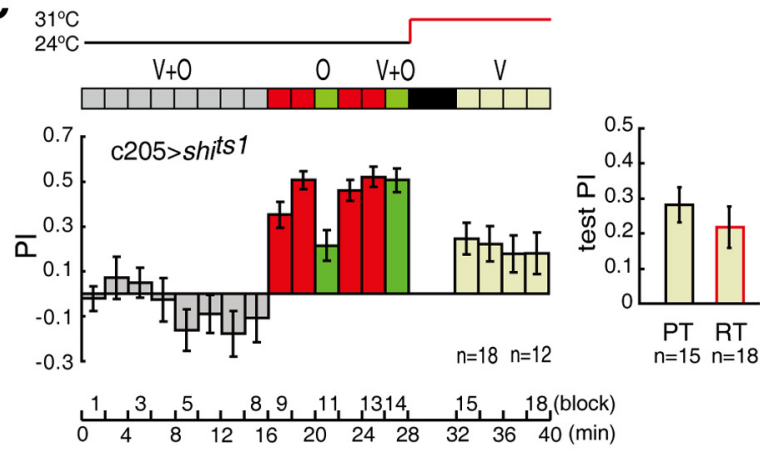

E

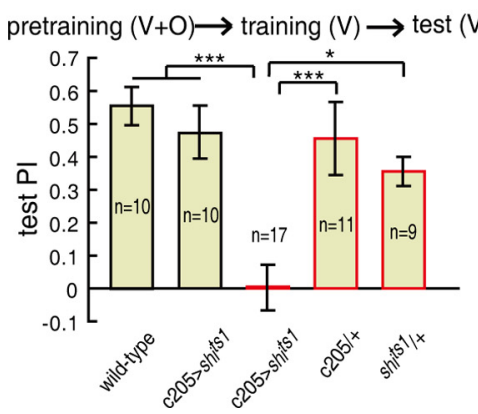

B



D
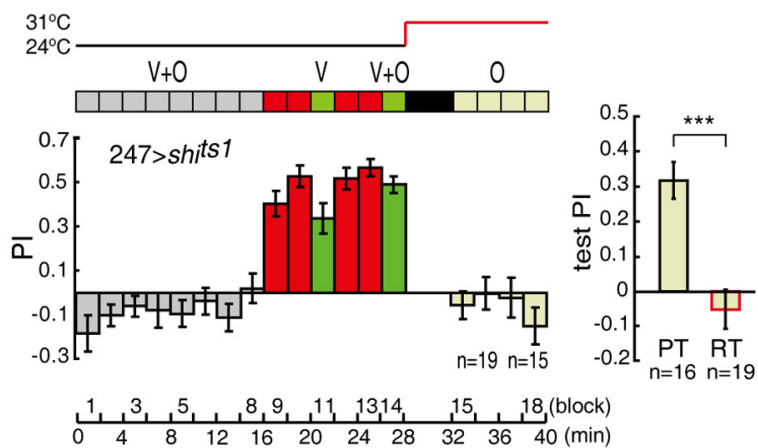

F



Figure 3. The conditioned response to CS1 can be recalled from parallel pathways. $\boldsymbol{A}-\boldsymbol{D}$, The cross-modal SP paradigms were similar to those in Figure 1, except that the test session was increased to $8 \mathrm{~min}$. Note that a portion of the flies failed to complete the lengthened trial with sustained flight. The colored blocks are defined as in Figure 1 . Each bar in the right panel represents the mean $\mathrm{PI}$ in the test session; the test Pls at the RT are represented by red rectangles, while test PIs at the PT are indicated by black rectangles. $A$, Blocking the synaptic output from the 247 neurons did not impair elevation memory retrieval in the olfaction $\rightarrow$ vision subsequence of the cross-modal SP. B, Blocking the synaptic output from the $c 205$ neurons during the test period did not affect odor memory retrieval in the vision $\rightarrow$ olfaction subsequence. $\boldsymbol{C}$, Blocking the synaptic output from the 205 neurons also did not affect elevation memory retrieval in the olfaction $\rightarrow$ vision subsequence. $\boldsymbol{D}$, Blocking the synaptic output from the 247 neurons impaired odor memory retrieval in the vision $\rightarrow$ olfaction subsequence. $\boldsymbol{E}, \boldsymbol{F}$, Each bar represents the mean PI in the test session. $\boldsymbol{E}$, Blocking the output from the 205 neurons indeed abolished elevation memory retrieval. $\boldsymbol{F}$, Control experiments for $\boldsymbol{D}$, indicating that memory retrieval impairment was not due to the RT. Error bars are $S$ EM. ${ }^{*} p \leq 0.05 ;{ }^{* * *} p \leq 0.001$.

section by shifting the $c 205>s h i^{t s 1}$ flies from the PT to the RT. The conditioned responses to the odors (CS1) were not compromised (Mann-Whitney test, $U=131, p=0.654$ ) (Fig. 3B). These results suggest that there may be parallel pathways for CS1 memory retrieval in the cross-modal SP paradigm.

If the parallel pathways contribute to memory retrieval compensatorily, blocking either one of the pathways would not affect memory retrieval. We further tested this hypothesis by blocking the F5 output during the test section in the olfaction $\rightarrow$ vision subsequence. Thus, we disrupted the potential CS1-US pathway by shifting the $c 205>s h i^{t s l}$ flies from the PT to the RT. As expected, memory retrieval was normal in the c205>shits $i^{t s}$ flies at the RT in the olfaction $\rightarrow$ vision subsequence (two-sample $t$ test, $t_{(31)}=0.828, p=0.414$ ) (Fig. $3 C$ ), indicating that there are two independent pathways for memory retrieval. However, elevation memory may also be restored from the ellipsoid body (Wang et al., 2008b). Therefore, we tested whether the output from F5 was necessary for elevation memory retrieval under the cross-modal SP paradigm. We demonstrated that blocking synaptic transmis- sion in F5 indeed abolished elevation memory retrieval $\left(\right.$ ANOVA, $F_{(4,52)}=10.661, p<0.001$ ) (Fig. 3E).

By contrast, olfactory memory retrieval could not be compensated if the CS1-US pathway was disrupted by blocking the MBs during the test section in the vision $\rightarrow$ olfaction subsequence. At the RT, 247>shis ${ }^{\text {tsl }}$ impaired the conditioned response to odors in the vision $\rightarrow$ olfaction subsequence (two-sample $t$ test, $t_{(33)}=$ 4.910, $p<0.001$ ) (Fig. 3D), but this impairment was not due to the RT itself (ANOVA, $F_{(2,40)}=16.344, p<0.001$ ) (Fig. 3F). This result suggests that both of the parallel pathways might depend on the outputs of the MBs and that the MBs may also play a role in the olfaction-vision-US pathway (CS1-CS2-US), particularly in the olfaction-vision association portion of this pathway.

\section{The MB $\boldsymbol{\alpha} / \boldsymbol{\beta}$ neurons are necessary for retrieving the} odor-elicited conditioned response of cross-modal SP The impaired memory observed above suggests that the MBs might be involved in the cross-modal SP. However, the olfactory memory-retrieval process and olfaction-vision association can 


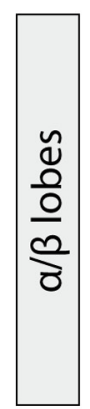

A


C
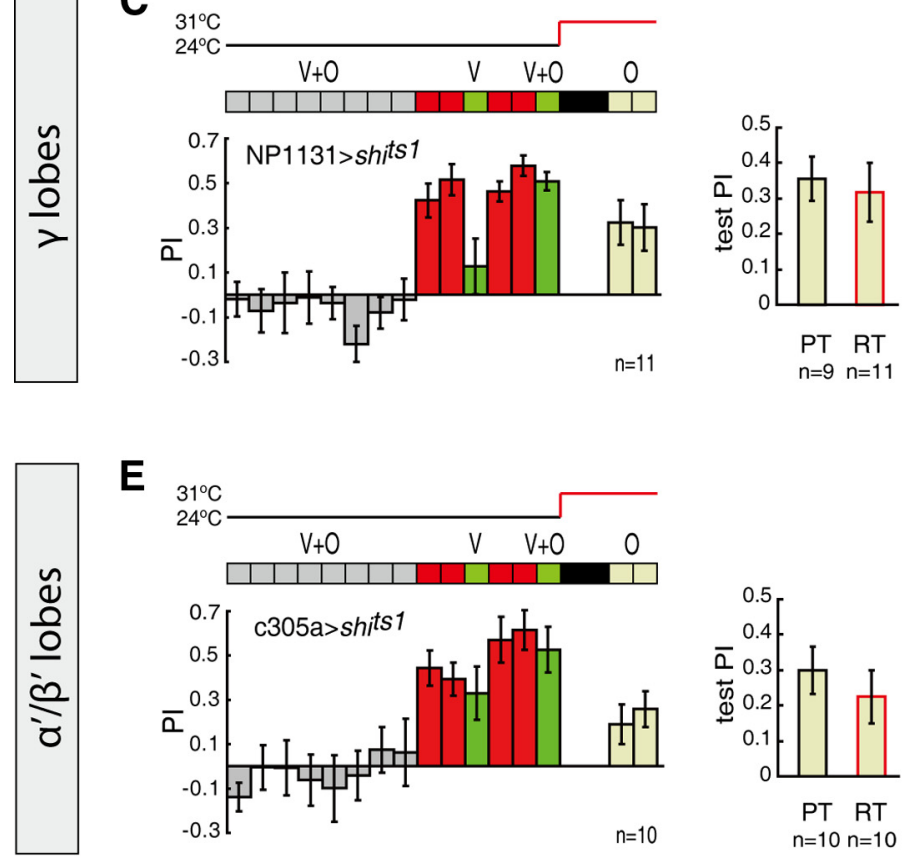

$\mathbf{E}$
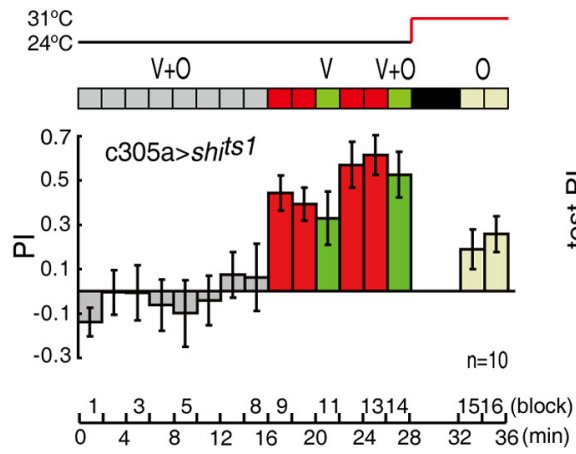

B
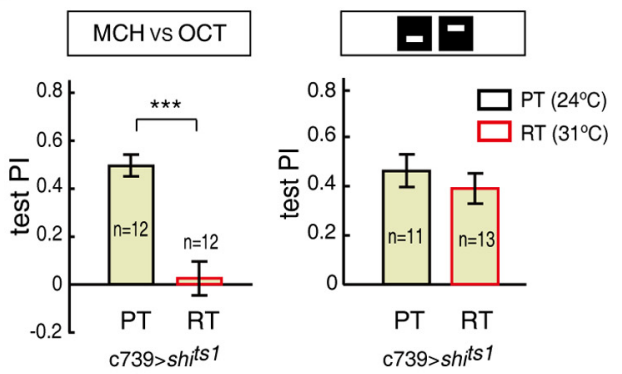

D
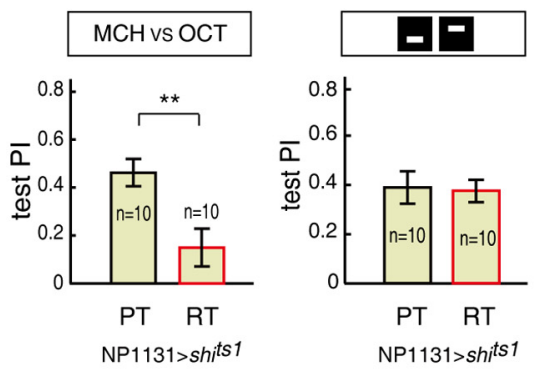

$\mathbf{F}$
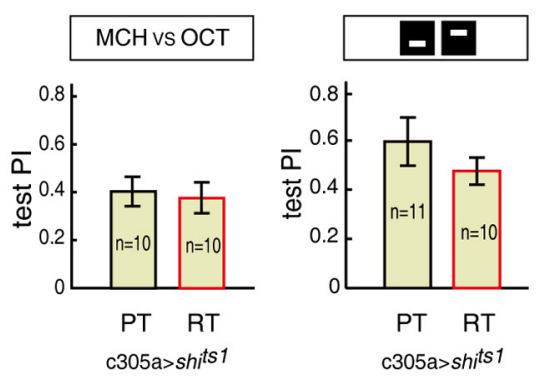

Figure 4. $\mathrm{MB} \alpha / \beta$ neurons are required for recalling the conditioned response to odors in cross-modal SP. $\boldsymbol{A}$, Blocking synaptic output from c739 $\alpha / \beta$ Gal4-labeled neurons impaired odor memory retrieval in the vision $\rightarrow$ olfaction subsequence of cross-modal SP. $B$, Blocking the output from c739 neurons impaired odor memory retrieval of unimodal olfactory conditioning (left) but did not affect unimodal visual memory retrieval (right). C, By contrast, blocking synaptic output from NP1131 $\gamma$ Gal4-labeled neurons did not affect odor memory retrieval in the vision $\rightarrow$ olfaction subsequence. D, Blocking output from NP1131 neurons impaired unimodal odor memory retrieval (left) but did not affect unimodal visual memory retrieval (right). $\boldsymbol{E}$, Blocking synaptic output from c305a $\alpha^{\prime} / \beta^{\prime}$ Gal4-labeled neurons did not affect odor memory retrieval in the vision $\rightarrow$ olfaction subsequence. $\boldsymbol{F}$, Blocking the output from c305a neurons did not significantly affect either unimodal odor memory retrieval or unimodal visual memory retrieval. Error bars are SEM. ${ }^{* *} p \leq 0.01 ;{ }^{* * *} p \leq 0.001$.

depend on the different subsets of the MB neurons, which include three major cell subtypes: the $\alpha / \beta, \alpha^{\prime} / \beta^{\prime}$, and $\gamma$ neurons. The axons of these neurons project to form vertical lobes ( $\alpha$ and $\alpha^{\prime}$ ) and horizontal lobes $(\beta, \beta$, and $\gamma$ ) (Strausfeld et al., 1998; Tanaka et al., 2008). To further dissect the role of the MBs in the crossmodal SP, we targeted $s h i^{t s l}$ expression to each cell type with MB lobe-specific Gal4 drivers. Specifically, we used c739, NP1131, and c305a, each of which yields expression in the $\alpha / \beta, \gamma$, and $\alpha^{\prime} / \beta^{\prime}$ neurons, respectively (Yang et al., 1995; Tanaka et al., 2008; Aso et al., 2009).

We tested these lines in the vision $\rightarrow$ olfaction subsequence of the cross-modal SP. Remarkably, the conditioned response to odors was impaired by the blockade of synaptic transmission in the $\alpha / \beta$ neurons (two-sample $t$ test, $t_{(17)}=4.985, p<0.001$ ) (Fig. $4 A$ ), which mimicked the phenotype of the $247>\operatorname{shi} i^{t s 1}$ group (Fig. 3D). Moreover, blocking the $\alpha / \beta$ neurons in the unimodal control experiments abolished the olfactory memory-retrieval process (two-sample $t$ test, $t_{(22)}=5.895, p<0.001$ ) but left the visual memory-retrieval process intact (two-sample $t$ test, $t_{(22)}=0.861, p=0.398$ ) (Fig. $4 B$ ). The results above suggest the involvement of the $\alpha / \beta$ neurons in both the olfaction-US and olfaction-vision associations.

However, blocking the output from $\gamma$ neurons did not impair the conditioned response to odors in the vision $\rightarrow$ olfaction subsequence (two-sample $t$ test, $t_{(18)}=0.370, p=0.716$ ) (Fig. $4 C$ ), although $\gamma$ neurons were necessary for the unimodal olfactory memory-retrieval process (two-sample $t$ test, $t_{(18)}=3.405, p=$ 0.003 ) (Fig. $4 D$, left). Therefore, when the olfaction-US pathway was blocked, the conditioned response to the odors could still be recalled through the olfaction-vision-US pathway in the $\gamma$-neuron dysfunction line. These results also indicate that the two pathways can compensate each other for CS1-elicited performance.

In addition, blocking the $\alpha^{\prime} / \beta^{\prime}$ neurons did not affect the olfactory memory-retrieval process in the vision $\rightarrow$ olfaction subsequence (two-sample $t$ test, $t_{(18)}=0.791, p=0.439$ ) (Fig. $4 E$ ). 



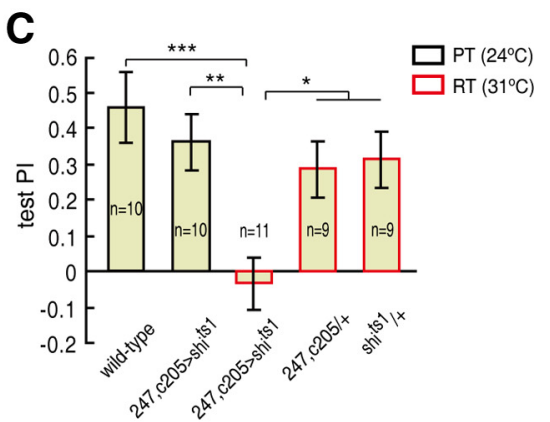

E

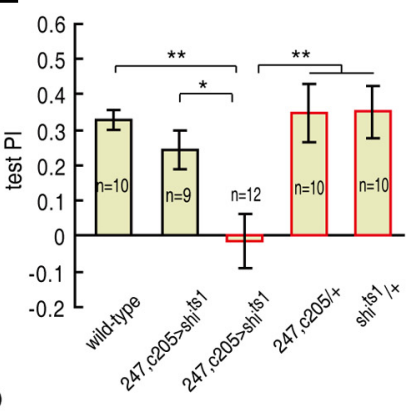

Figure 5. Blocking MB and F5 output simultaneously abolished memory retrieval in cross-modal SP. A, Gal4-driven GFP expression in the three driver lines. Ai-iii, The GFP signal (green) and the specimen labeled by the anti-nc82 antibody (magenta) were excited at 488 and $561 \mathrm{~nm}$, respectively. The (247,c205)-Gal4 labeled both MBs and F5. All images were superimposed confocal sections. FB, Fan-shaped body. Scale bars: $50 \mu \mathrm{m}$. B, D, Blocking synaptic output from (247,c205)-Gal4 neurons impaired memory retrieval in both the olfaction $\rightarrow$ vision and vision $\rightarrow$ olfaction subsequences of cross-modal SP paradigms at the RT. $\boldsymbol{C}, \boldsymbol{E}$, Control experiments for $\boldsymbol{B}$ and $\boldsymbol{D}$, respectively; each bar represents the mean PI in the test section. Error bars are SEM. ${ }^{*} p \leq 0.05$; ${ }^{* *} p \leq$ $0.01 ;{ }^{* *} p \leq 0.001$.

The $\alpha^{\prime} / \beta^{\prime}$ neurons were not necessary for the unimodal olfactory memory-retrieval process (two-sample $t$ test, $t_{(18)}=0.313, p=$ 0.758 ) (Fig. $4 F$, left). Although the visual memory retrieval was slightly impaired by the blockade of synaptic transmission in the c305a line (Mann-Whitney test, $U=28, p=0.061$ ) (Fig. $4 F$, right), the PI was still significantly higher than that due to chance (one-sample $t$ test, $t_{(9)}=9.084, p<0.001$ ). Thus, the results observed for the drivers from the distinct MB cell types suggest that the $\alpha / \beta$ but not the $\gamma$ neurons may play a role in the olfaction-vision association in the memory-retrieval process of crossmodal SP.

Blocking MB and F5 output simultaneously abolishes the memory retrieval of cross-modal SP

Finally, we generated the (247, c205)-Gal4 line by recombining the 247 and c205 lines to achieve both expression patterns (Fig. $5 A$ ). Thus, the output of the MBs and F5 can be blocked simultaneously via expression of $s h i^{t s 1}$ in $(247$, c205) - Gal4. The conditioned responses to the unreinforced cues were impaired in the $(247, \mathrm{c} 205)>s i^{t s l}$ flies at the RT during the test session in both the olfaction $\rightarrow$ vision and the vision $\rightarrow$ olfaction subsequences $\left(\right.$ ANOVA, $F_{(4,44)}=6.169, p<0.001$ and $F_{(4,46)}=6.227, p<$ 0.001 , respectively) (Fig. $5 B-E$ ). These conditioned responses were totally abolished by the impairment, as indicated by the PIs, which were not significantly different from zero (one-sample $t$ test, $t_{(10)}=-0.482, p=0.640$ and $t_{(11)}=-0.209, p=0.838$, respectively). These results, together with the normal conditioned responses shown in Figure 3, $A$ and $B$, support the existence of parallel pathways for memory-retrieval processing.

\section{Discussion}

In the cross-modal SP paradigm, one conditioned stimulus (CS1) initially paired with another conditioned stimulus (CS2) from a different sensory modality could trigger a conditioned response after pairing of CS2 with a US (Fig. $6 A$ ). The prevailing hypothesis is that the conditioned response to CS1 relies on the CS2 memory via a CS1-CS2 association, which suggests a shared neural pathway for memory-retrieval of both CS1 and CS2 (Fig. 6B, solid arrows). Here, using a flight simulator with fruit flies, we adopted the strategy of specifically disrupting the CS2-US memory retrieval to test whether other pathways exist for expressing the conditioned response to CS1. Under the vision-olfaction cross-modal SP paradigm, our results demonstrated that fruit flies could still recall the CS1 memory after CS2 memory retrieval had been fully disrupted, regardless of the modality of CS2. This finding indicates the formation of another memory trace (e.g., the CS1-US association indicated by the dashed arrow in Fig. 6 B) and suggests separated neural structures or parallel pathways for CS1 memory-retrieval processing.

The cross-modal SP paradigm used here (Fig. 1) was slightly different from that of our previous study (Guo and Guo, 2005). First, instead of the totally dark background, we randomly covered $2 \%$ of the area of the panorama with white dots. We demonstrated that the random dots did not disturb the CS conditioning or memory retrieval (Fig. $1 D, E$ ). This finding was consistent with a previous study that demonstrated that olfactory conditioning cannot be altered by visual context in fruit flies (Yarali et al., 2008). Second, by shifting the ambient temperature from $24^{\circ} \mathrm{C}(\mathrm{PT})$ to $31^{\circ} \mathrm{C}(\mathrm{RT})$ in subsequent experiments, a dark period before the final test session was added. However, the test performance became unstable after the interval interference, resulting in memory decay (data not shown). Interestingly, the addition of a 2 min reminder block before the dark period induced a robust conditioned response to the unreinforced cues (Fig. $1 B, C$ ) rather than causing memory extinc- 
A



(2)

C

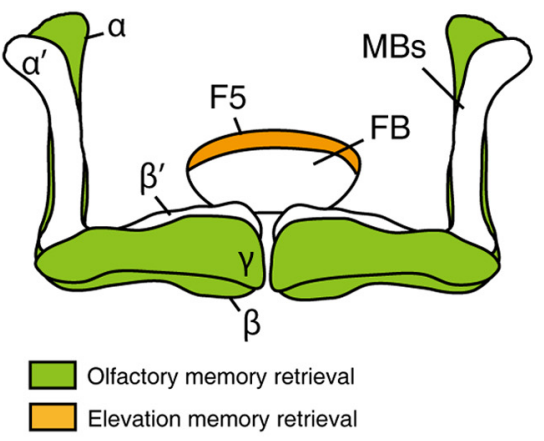

D

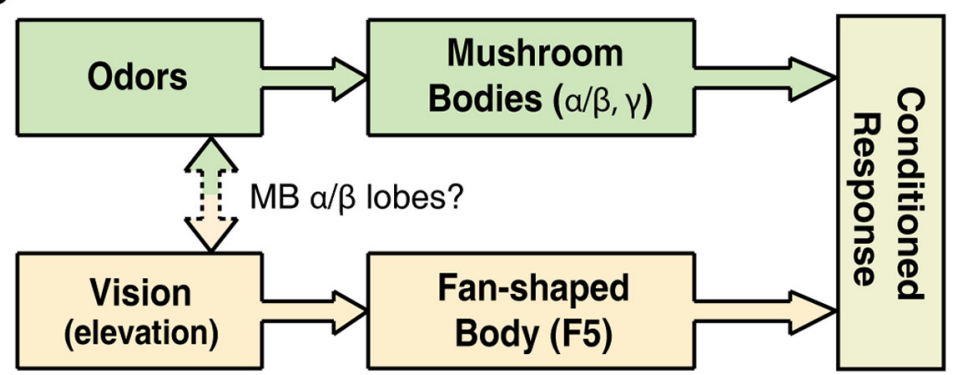

Figure 6. A model of memory retrieval processing of cross-modal SP. $\boldsymbol{A}$, The procedure of cross-modal SP. $\boldsymbol{B}, \mathrm{A}$ schematic representation of hypothetical associations that could be formed for memory retrieval processing of cross-modal SP. The solid arrows indicate the pathway of the former model for CS1 memory retrieval; the dashed arrow represents another hypothetical pathway for CS1 memory retrieval. C, Anatomical representation of unimodal memory retrieval. Separated neural structures for olfactory and visual elevation memory retrieval: the $\alpha / \beta$ and $\gamma$ lobes of MBs are necessary for olfactory memory retrieval; F5 is necessary for elevation memory retrieval. $D$, A model of a circuit for memory retrieval of cross-modal SP. Two memory traces are formed after CS2 conditioning, each of which can be retrieved independently. The two pathways could correspond through the olfaction-vision association, which may require the $\alpha / \beta$ lobes of MBs. CR, Conditioned response; FB, fan-shaped body.

the visual cues (Fig. 3A). These data suggest that flies can recall the memory from the vision-US association directly (Fig. $6 B$, dashed arrow). In addition, we blocked the F5 neurons during the vision $\rightarrow$ olfaction subsequence and found that flies also exhibited normal olfactory memory performance. Therefore, these results indicate that two memory traces have been constructed for CS1 memory retrieval using parallel pathways (Fig. $6 D)$. Moreover, we disrupted the potential CS1-US association memory retrieval in the cross-modal SP paradigms, which revealed that blocking the F5 neurons in the test section of the olfaction $\rightarrow$ vision subsequence did not impair the conditioned response to the visual cues (Fig. 3C) and that blocking the MBs $\gamma$ neurons in the vision $\rightarrow$ olfaction subsequence did not affect the conditioned response to the odors (Fig. 4C). Thus, these results suggest that the two pathways can compensate for each other and that the maintenance of only one of these pathways is sufficient for memory retrieval (Fig. 6D). Interestingly, a recent study suggested that only the CS1-US pathway, but not the CS1-CS2 association pathway, is required for long-term memory recall of cross-modal SP in crickets (Matsumoto et al., 2013).

Remarkably, memory retrieval was not compensated for when the prime neurons tion or a second-order conditioning effect (Tabone and de Belle, 2011).

In Drosophila, specific memory traces have been mapped to specific neuroanatomical structures (Zars, 2010). We used the 247 and c205 Gal4 drivers to block the synaptic output of the prime neurons of the MBs (Krashes et al., 2007; Tan et al., 2010) and F5 neurons of the fan-shaped body (F5) (Liu et al., 2006; Li et al., 2009), respectively. We found that blocking the output of the prime neurons of the MBs specifically abolished the olfactory memory-retrieval process of the fly in the flight simulator (Fig. 2B). This result was largely consistent with previous studies conducted in the T-maze (Dubnau et al., 2001; McGuire et al., 2001). In addition, we used MB lobes-specific Gal4 drivers and found that blocking either the $\alpha / \beta$ or $\gamma$ neurons fully disrupted the odor memory-retrieval process (Fig. $4 B, D$ ). By contrast, blocking the $\alpha^{\prime} / \beta^{\prime}$ neurons did not affect the odor memory-retrieval process (Fig. $4 F$ ). These results are supported by previous studies in both larval and adult flies (Krashes et al., 2007; Honjo and FurukuboTokunaga, 2009; Pauls et al., 2010). We also found that blocking the output from F5 neurons specifically abolished the visual elevation memory-retrieval process (Fig. $2 C$ ). Thus, we demonstrated that the outputs of the MBs ( $\alpha / \beta$ and $\gamma$ neurons) and F5 are necessary for unimodal olfactory and visual elevation memory retrieval, respectively (Fig. 6C).

Then, we blocked either memory modality from the crossmodal SP paradigm by double dissociation. In the olfaction $\rightarrow$ vision subsequence of the SP paradigm, only the olfactory cues were reinforced. We blocked the prime neurons of the MBs and found that the flies still exhibited robust conditioned responses to of the MBs were blocked in the vision $\rightarrow$ olfaction subsequence (Fig. 3D); we assumed that blocking the output of MBs cutoff both pathways for memory retrieval in the vision $\rightarrow$ olfaction subsequence, as blocking both MBs and F5 did (Fig. 5D). In this case, the flies could not recall the memories of odors through either the olfaction-vision-US pathway or the olfaction-US pathway. These data suggest that MBs may be also involved in the process of forming the olfaction-vision association (Fig. 6D). The involvement of the MBs was further dissected by using MB lobe-specific Gal4 drivers (Fig. 4). We found that $\alpha / \beta$ neurons but not $\gamma$ neurons may be involved in the olfaction-vision association process (Fig. $4 A-D$ ). However, whether the $\alpha^{\prime} / \beta^{\prime}$ neurons are necessary for the olfaction-vision association cannot be deduced from these experiments (Fig. $4 E, F$ ) because the blockade of synaptic transmission in the $\alpha^{\prime} / \beta^{\prime}$ neurons did not impair the unimodal olfactory memory retrieval (Fig. $4 F$ ).

The interpretation that the $\alpha / \beta$ neurons of the MBs are necessary for the olfaction-vision association was also supported by the observations that the MBs played a role in multi-sensory integration (Li and Strausfeld, 1999; Balkenius et al., 2009; Chow et al., 2011). In addition, the MBs were reported to play complicated roles in visual tasks, including contextual generalization (Liu et al., 1999; Brembs and Wiener, 2006), attention (Xi et al., 2008; van Swinderen et al., 2009), decision making (Tang and Guo, 2001; Zhang et al., 2007), and behavioral flexibility (Brembs, 2009; Ren et al., 2012). However, unlike honeybees (Ehmer and Gronenberg, 2002), there is little anatomical evidence for a direct connection between the visual pathway and the MBs in Drosophila. These interactions may be achieved indirectly 
via other regions (e.g., the ventrolateral protocerebrum) that receive projection neurons from different modalities (Tanaka et al., 2004; Kamikouchi et al., 2006; Otsuna and Ito, 2006) or via synchronized activity between modality-specific nuclei (van Swinderen and Greenspan, 2003).

Taken together, our data demonstrate parallel pathways for memory retrieval in the cross-modal SP paradigm in Drosophila. Our results suggest that memory traces of each modality exist in dedicated neural circuits and that either memory can be retrieved via a CS1-CS2 association (Fig. 6D). However, an alternative hypothesis suggests that there may be a "configural unit"-stimulus association for memory retrieval (Hall and Suboski, 1995), in which visual and olfactory cues are combined as a compound conditioned stimulus that can be activated by either visual or olfactory cues via their similarity to the compound conditioned stimulus. Consequently, conditioning with CS2 would form not only the CS2-US but also the configural unit-US association. Thus, the conditioned response to CS1 could be recalled via the similarity between CS1 and the compound conditioned stimulus, and thus, the CS1-US association would be unnecessary for the CS1-elicited response in this case. This hypothesized pathway requires the involvement of another brain structure that would hold the cross-modal configural unit-US association.

However, our results did not support the "configural unit hypothesis.” In the cross-modal SP, blocking either MB neurons or F5 neurons did not disrupt the retrieval of the configural unit memory trace (Fig. $3 A, B$ ). These results indicate that additional neural circuits should be recruited for the configural unit-US association, if the configural unit hypothesis is correct. Therefore, simultaneously blocking MBs and F5 would also not affect the conditioned response to CS1. On the contrary, our results indicated that blocking both the MB neurons and the F5 neurons abolished the CS1 elicited response (Fig. 5). Thus, it is more likely that the CS1-US association, but not the configural unit-US association is required for cross-modal memory retrieval. However, when and how the potential CS1-US association is acquired requires further investigation. Moreover, other SP paradigms that include the intramodal interactions are also expected to be validated based on the understanding developed in the present study of the circuit mechanisms underlying higher-order conditioning selected in the natural environment.

\section{References}

Aso Y, Grübel K, Busch S, Friedrich AB, Siwanowicz I, Tanimoto H (2009) The mushroom body of adult Drosophila characterized by GAL4 drivers. J Neurogenet 23:156-172. CrossRef Medline

Balkenius A, Bisch-Knaden S, Hansson B (2009) Interaction of visual and odour cues in the mushroom body of the hawkmoth Manduca sexta. J Exp Biol 212:535-541. CrossRef Medline

Brembs B (2009) Mushroom Bodies Regulate Habit Formation in Drosophila. Curr Biol 19:1351-1355. CrossRef Medline

Brembs B, Heisenberg M (2001) Conditioning with compound stimuli in Drosophila melanogaster in the flight simulator. J Exp Biol 204:28492859. Medline

Brembs B, Wiener J (2006) Context and occasion setting in Drosophila visual learning. Learn Mem 13:618-628. CrossRef Medline

Brogden WJ (1939) Sensory pre-conditioning. J Exp Psychol 25:323-332. CrossRef

Chow DM, Theobald JC, Frye MA (2011) An olfactory circuit increases the fidelity of visual behavior. J Neurosci 31:15035-15047. CrossRef Medline

Davis RL (2011) Traces of Drosophila memory. Neuron 70:8-19. CrossRef Medline

Dubnau J, Grady L, Kitamoto T, Tully T (2001) Disruption of neurotransmission in Drosophila mushroom body blocks retrieval but not acquisition of memory. Nature 411:476-480. CrossRef Medline
Duistermars BJ, Frye MA (2008) Crossmodal visual input for odor tracking during fly flight. Curr Biol 18:270-275. CrossRef Medline

Ehmer B, Gronenberg W (2002) Segregation of visual input to the mushroom bodies in the honeybee (Apis mellifera). J Comp Neurol 451:362373. CrossRef Medline

Gewirtz JC, Davis M (2000) Using Pavlovian higher-order conditioning paradigms to investigate the neural substrates of emotional learning and memory. Learn Mem 7:257-266. CrossRef Medline

Götz KG (1964) Optomotorische Untersuchung des visuellen systems einiger Augenmutanten der Fruchtfliege Drosophila. Kybernetik 2:77-92. CrossRef Medline

Guo A, Li L, Xia SZ, Feng CH, Wolf R, Heisenberg M (1996) Conditioned visual flight orientation in Drosophila: dependence on age, practice, and diet. Learn Mem 3:49-59. CrossRef Medline

Guo J, Guo A (2005) Crossmodal interactions between olfactory and visual learning in Drosophila. Science 309:307-310. CrossRef Medline

Hall D, Suboski MD (1995) Sensory preconditioning and second-order conditioning of alarm reactions in zebra danio fish (Brachydanio rerio). J Comp Psychol 109:76-84. CrossRef

Heisenberg M (2003) Mushroom body memoir: from maps to models. Nat Rev Neurosci 4:266-275. CrossRef Medline

Honjo K, Furukubo-Tokunaga K (2009) Distinctive neuronal networks and biochemical pathways for appetitive and aversive memory in Drosophila larvae. J Neurosci 29:852-862. CrossRef Medline

Kamikouchi A, Shimada T, Ito K (2006) Comprehensive classification of the auditory sensory projections in the brain of the fruit fly Drosophila melanogaster. J Comp Neurol 499:317-356. CrossRef Medline

Kitamoto T (2001) Conditional modification of behavior in Drosophila by targeted expression of a temperature-sensitive shibire allele in defined neurons. J Neurobiol 47:81-92. CrossRef Medline

Kojima S, Kobayashi S, Yamanaka M, Sadamoto H, Nakamura H, Fujito Y, Kawai R, Sakakibara M, Ito E (1998) Sensory preconditioning for feeding response in the pond snail, Lymnaea stagnalis. Brain Res 808:113-115. CrossRef Medline

Krashes MJ, Keene AC, Leung B, Armstrong JD, Waddell S (2007) Sequential use of mushroom body neuron subsets during Drosophila odor memory processing. Neuron 53:103-115. CrossRef Medline

Li W, Pan Y, Wang Z, Gong H, Gong Z, Liu L (2009) Morphological characterization of single fan-shaped body neurons in Drosophila melanogaster. Cell Tissue Res 336:509-519. CrossRef Medline

Li Y, Strausfeld NJ (1999) Multimodal efferent and recurrent neurons in the medial lobes of cockroach mushroom bodies. J Comp Neurol 409:647663. CrossRef Medline

Liu G, Seiler H, Wen A, Zars T, Ito K, Wolf R, Heisenberg M, Liu L (2006) Distinct memory traces for two visual features in the Drosophila brain. Nature 439:551-556. CrossRef Medline

Liu L, Wolf R, Ernst R, Heisenberg M (1999) Context generalization in Drosophila visual learning requires the mushroom bodies. Nature 400:753756. CrossRef Medline

Matsumoto Y, Hirashima D, Mizunami M (2013) Analysis and modeling of neural processes underlying sensory preconditioning. Neurobiol Learn Mem 101:103-113. CrossRef Medline

McGuire SE, Le PT, Davis RL (2001) The role of Drosophila mushroom body signaling in olfactory memory. Science 293:1330-1333. CrossRef Medline

Müller D, Gerber B, Hellstern F, Hammer M, Menzel R (2000) Sensory preconditioning in honeybees. J Exp Biol 203:1351-1364. Medline

Nicholson DA, Freeman JH Jr (2000) Lesions of the perirhinal cortex impair sensory preconditioning in rats. Behav Brain Res 112:69-75. CrossRef Medline

Otsuna H, Ito K (2006) Systematic analysis of the visual projection neurons of Drosophila melanogaster. I. Lobula-specific pathways. J Comp Neurol 497:928-958. CrossRef Medline

Pauls D, Selcho M, Gendre N, Stocker RF, Thum AS (2010) Drosophila larvae establish appetitive olfactory memories via mushroom body neurons of embryonic origin. J Neurosci 30:10655-10666. CrossRef Medline

Ren Q, Li H, Wu Y, Ren J, Guo A (2012) A GABAergic inhibitory neural circuit regulates visual reversal learning in Drosophila. J Neurosci 32: 11524-11538. CrossRef Medline

Robinson S, Keene CS, Iaccarino HF, Duan D, Bucci DJ (2011) Involvement of retrosplenial cortex in forming associations between multiple sensory stimuli. Behav Neurosci 125:578-587. CrossRef Medline 
Strausfeld NJ, Hansen L, Li Y, Gomez RS, Ito K (1998) Evolution, discovery, and interpretations of arthropod mushroom bodies. Learn Mem 5:11-37. Medline

Tabone CJ, de Belle JS (2011) Second-order conditioning in Drosophila. Learn Mem 18:250-253. CrossRef Medline

Tait RW, Suboski MD, Black M, Katz M (1972) Discriminative sensory preconditioning. Can J Psychology 26:201-205. CrossRef

Tan Y, Yu D, Pletting J, Davis RL (2010) Gilgamesh is required for rutabagaindependent olfactory learning in Drosophila. Neuron 67:810-820. CrossRef Medline

Tanaka NK, Awasaki T, Shimada T, Ito K (2004) Integration of chemosensory pathways in the Drosophila second-order olfactory centers. Curr Biol 14:449-457. CrossRef Medline

Tanaka NK, Tanimoto H, Ito K (2008) Neuronal assemblies of the Drosophila mushroom body. J Comp Neurol 508:711-755. CrossRef Medline

Tang S, Guo A (2001) Choice behavior of Drosophila facing contradictory visual cues. Science 294:1543-1547. CrossRef Medline

van Swinderen B, Greenspan RJ (2003) Salience modulates 20-30 Hz brain activity in Drosophila. Nat Neurosci 6:579-586. CrossRef Medline

van Swinderen B, McCartney A, Kauffman S, Flores K, Agrawal K, Wagner J, Paulk A (2009) Shared visual attention and memory systems in the Drosophila brain. PLoS One 4:e5989. CrossRef Medline

Wang Y, Mamiya A, Chiang AS, Zhong Y (2008a) Imaging of an early memory trace in the Drosophila mushroom body. J Neurosci 28:4368-4376. CrossRef Medline
Wang Z, Pan Y, Li W, Jiang H, Chatzimanolis L, Chang J, Gong Z, Liu L (2008b) Visual pattern memory requires foraging function in the central complex of Drosophila. Learn Mem 15:133-142. CrossRef Medline

Wolf R, Heisenberg M (1991) Basic organization of operant behavior as revealed in Drosophila flight orientation. J Comp Physiol A 169:699_ 705. Medline

Wu JS, Luo L (2006) A protocol for dissecting Drosophila melanogaster brains for live imaging or immunostaining. Nat Protoc 1:2110-2115. CrossRef Medline

Xi W, Peng Y, Guo J, Ye Y, Zhang K, Yu F, Guo A (2008) Mushroom bodies modulate salience-based selective fixation behavior in Drosophila. Eur J Neurosci 27:1441-1451. CrossRef Medline

Yang MY, Armstrong JD, Vilinsky I, Strausfeld NJ, Kaiser K (1995) Subdivision of the Drosophila mushroom bodies by enhancer-trap expression patterns. Neuron 15:45-54. CrossRef Medline

Yarali A, Mayerle M, Nawroth C, Gerber B (2008) No evidence for visual context-dependency of olfactory learning in Drosophila. Naturwissenschaften 95:767-774. CrossRef Medline

Zars T (2010) Short-term memories in Drosophila are governed by general and specific genetic systems. Learn Mem 17:246-251. CrossRef Medline

Zhang K, Guo JZ, Peng Y, Xi W, Guo A (2007) Dopamine-mushroom body circuit regulates saliency-based decision-making in Drosophila. Science 316:1901-1904. CrossRef Medline 